\title{
Directed evolution of the PcaV allosteric transcription factor to generate a biosensor for aromatic aldehydes
}

\author{
Leopoldo F. M. Machado ${ }^{1,2}$, Andrew Currin ${ }^{1,2,3}$ and Neil Dixon ${ }^{1,2,3^{*}}$ (B)
}

\begin{abstract}
Background: Transcription factor-based biosensors are useful tools for the detection of metabolites and industrially valuable molecules, and present many potential applications in biotechnology and biomedicine. However, the most common approach to develop biosensors relies on employing a limited set of naturally occurring allosteric transcription factors (aTFs). Therefore, altering the ligand specificity of aTFs towards the detection of new effectors is an important goal.

Results: Here, the PcaV repressor, a member of the MarR aTF family, was used to develop a biosensor for the detection of hydroxyl-substituted benzoic acids, including protocatechuic acid (PCA). The PCA biosensor was further subjected to directed evolution to alter its ligand specificity towards vanillin and other closely related aromatic aldehydes, to generate the Van2 biosensor. Ligand recognition of Van2 was explored in vitro using a range of biochemical and biophysical analyses, and extensive in vivo genetic-phenotypic analysis was performed to determine the role of each amino acid change upon biosensor performance.

Conclusions: This is the first study to report directed evolution of a member of the MarR aTF family, and demonstrates the plasticity of the PCA biosensor by altering its ligand specificity to generate a biosensor for aromatic aldehydes.
\end{abstract}

\section{Background}

Biosensing is an essential mechanism widely employed in nature to perceive environment conditions; such as the presence of certain intracellular or extracellular metabolites; nutrients, metal ions and stress signals $[1,2]$. Sensing is commonly associated with the regulation of gene expression at the transcriptional level mediated by an allosteric transcription factor (aTF). The aTF is a protein composed of an effector binding domain (EBD) that senses an effector molecule and a DNA binding domain (DBD) that interacts with a specific DNA operator sequence. Binding of an effector to the EBD leads to an allosterically induced conformation change affecting the interaction of the DBD with the DNA, hence modulating promoter

\footnotetext{
* Correspondence: neil.dixon@manchester.ac.uk

${ }^{1}$ Manchester Institute of Biotechnology (MIB), The University of Manchester,

M1 7DN, Manchester, UK

${ }^{2}$ Department of Chemistry, The University of Manchester, M1 7DN,

Manchester, UK

Full list of author information is available at the end of the article
}

accessibility and transcription initiation [1, 3, 4]. Generally, aTFs can be divided into repressors or activators. Repressors bind to a DNA operator blocking the RNA polymerase (RNAP) promoter binding, subsequent effector binding to the EBD leads to derepression allowing transcription initiation. Activators bind to DNA operator sequences permitting RNAP binding to the promoter to active transcription, often requiring aTF contact with the RNAP $\alpha$-subunit $[3,4]$.

An aTF and operator sequence can be harnessed and used in the design of genetic circuits to control expression of a reporter gene, generating a genetically encoded biosensor for the cognate effector [5, 6]. The reporter is usually a fluorescent protein, an enzyme or a toxic gene allowing a range of applications including high-throughput screening methods [7], adaptive laboratory evolution [8, 9], enzyme directed evolution [10-13], metabolic pathway optimisation [14-17] and metabolite monitoring [18]. Gene regulation tools based on aTFs have revolutionised biology. 
Canonical aTFs like LacI [19], AraC [20] and TetR [21] have been applied as inducible gene expression for decades. More recently, aTFs have been employed as biosensors for the detection of other molecules including industrially valuable aliphatic $[14,22,23]$ and aromatic [24-26] chemicals. Although aTF biosensors have been broadly used, the repertoire of effectors with a known aTF is limited [6]. Therefore strategies to generate new biosensors for desired molecules have been pursued $[27,28]$.

The directed evolution of the aTF to change its ligand specificity is a versatile alternative to develop new biosensors [29-34]. Nonetheless, engineering transcription factors is inherently challenging due to their allosteric nature. Small changes in the EBD can affect the stability of the DBD and the performance of the whole system [35]. Examples of aTFs reengineered to accommodate alternative ligands include LacI $[31,36]$, TetR $[37,38]$, AraC [30, 39, 40] and TrpR [34]. In general, these systems are very well characterised biochemically, structurally, and mechanistically, which greatly facilitates the directed evolution process. In addition, less well characterised systems such as PobR were reengineered with support of computational methods [32, 33].

$\mathrm{PcaV}$ is an aTF repressor of the MarR family involved in the genetic regulation of the catabolic genes for protocatechuic acid (PCA) in Streptomyces coelicolor $[41,42]$. This family of aTFs include examples for the sensing of environmental signals including antibiotics, toxic molecules, reactive species [43] and small molecule metabolites [44]. In contrast to most of the aTFs previously employed in directed evolution studies, relatively less is known about the allosteric mechanism of the MarR aTFs [45, 46].

Aromatic aldehydes are important molecules in an industrial context, as they can be used as chemical building blocks and have major applications in the flavoring industry, especially benzaldehyde and vanillin $[47,48]$. Furthermore, some aromatic aldehydes can be produced from lignin, a major component of plant biomass, therefore providing an alternative sustainable source for these key chemical building blocks away from fossil-derived sources [49]. The presence of a large market added to low yields from natural extraction and negative perception towards synthetic chemistry methods have recently increased the demand for biotechnological production of aromatic aldehydes $[50,51]$. Therefore, improvements in the bioproduction and application of these molecules would be highly desirable and valuable, and would be greatly enhanced by the development of new biosensor systems to expand the biotechnological tools available for discovery and innovation in the area.

In this study a PCA biosensor, based on the PcaV aTF, was developed and a wide range of candidate effectors were assessed. A directed evolution strategy of PcaV was then employed to alter the ligand specificity of the PCA biosensor to detect a range of small aromatic aldehydes including vanillin. The resultant Van2 biosensor was functionally and biochemically characterised and further mutations were introduced to explore the role of each amino acid change upon the biosensor performance.

\section{Methods}

Primers, plasmids and strains

The DNA oligos used in this study for constructions and sequencing are presented in Additional file 1: Table S1; oligos used to generate the PcaV DE libraries are described in the Additional file 1: Table S2. Plasmids details are described in Additional file 1: Table S4. Strains are described in Additional file 1: Table S5. The DNA parts with the gene for $p c a V$ and the promoters $\mathrm{P}_{\mathrm{LV}}$ and $\mathrm{P}_{\mathrm{PV}}$ were synthetized by GenArt. pcaV was cloned into a p15 plasmid flanked by a $\mathrm{P}_{\mathrm{LacI}}$ constitutive promoter and a rrnB1 terminator by restriction digestion of the NdeI and XbaI sites. $\mathrm{P}_{\mathrm{LV}}$ and $\mathrm{P}_{\mathrm{PV}}$ were cloned into a pET44-eGFP plasmid [26] upstream to an eGFP gene by restriction digestion using NdeI and SphI sites. Cloning transformation was made into E. coli DH5-alpha (NEB), transformation for induction test was made into $E$. coli BL21 (NEB).

\section{Chemicals}

The chemicals used in this study with the description and suppliers are presented in Additional file 1: Tables S6 and S7. Stocks were prepared dissolving the chemicals in dimethyl sulfoxide (DMSO) at 100 $\mathrm{mM}$ concentration. Dilution of the stocks was made dissolving them in water, or DMSO for hydrophobic chemicals.

\section{Biosensor cultures growth and induction assays}

E. coli (BL21, BW25113 or RARE [52] strains) were cotransformed with the required plasmids for the PCA biosensor system, PcaV library (Additional file 1: Table S4) or Van2 biosensor system and grown in LB media supplemented with chloramphenicol $(30 \mu \mathrm{g} / \mathrm{mL})$ and ampicillin $(100 \mu \mathrm{g} / \mathrm{mL})$. The induction assays were performed by the addition of the tested potential ligands or effectors to the E. coli biosensor cultures freshly grown to exponential/mid-log stage (cell density OD 0.6) in deep well plates. The eGFP expression was monitored following 3 hours of induction at $37{ }^{\circ} \mathrm{C}$ with shaking at 1000 RPM. Cells were centrifuged, washed and re-suspended with PBS buffer. The expression output was then analysed by monitoring the fluorescence normalised to cell density (RFU/OD 600 ) in a plate reader (ClarioStar). Graphs were plotted using GraphPad PRISM 7 and the dose response was fitted with a Hill function. 


\section{PcaV libraries design and construction}

The PcaV libraries were constructed using the SpeedyGenes gene synthesis method [53, 54]. PcaV WT and library genes were designed with the GeneGenie software [55], allowing the generation of WT or variant libraries by overlap-extension PCR (OE-PCR) using 10 overlapping oligonucleotides. Terminal primers also encoded homologous cloning sequences to allow the ligation of the final PCR product into the p15 plasmid backbone. Wild type and variant (NNK codon) oligonucleotides were designed for each of the 7 selected amino acid positions, His9, His21, Trp25, Tyr38, Ile110, Met113, Asn114 (Additional file 1: Table S2). Different combinations of the oligos in the Additional file 1: Table S2 were then used to generate libraries of PcaV mutants completely randomized for 3 amino acids (libraries PcaV DE L1; L2; L3 and L4) (Additional file 1: Table S3).

PcaV WT and PcaV libraries were made in two steps. First an OE-PCR was performed employing the selected set of 10 oligonucleotides, according to the desired product. Amplification was made using a Q5 Hot-Start HighFidelity DNA Polymerase (NEB) with 35 cycles of Denaturation $\left(98^{\circ} \mathrm{C}, 20 \mathrm{~s}\right)$, Annealing $\left(65^{\circ} \mathrm{C}, 15 \mathrm{~s}\right)$ and $\mathrm{Ex}-$ tension $\left(72^{\circ} \mathrm{C}, 30 \mathrm{~s}\right)$. Secondly, the product band was gel purified with the MinElute Gel Extraction Kit (QIAGEN) and an endonuclease correction step was performed with the Surveyor ${ }^{\circ}$ Mutation Detection Kit (IDT) as previously described at [53]. Finally, a second PCR step was performed to amplify the Surveyor treated template with the gene's end primers (PcaV DE WT 1F and PcaV DE WT 10R). The final PCR product was cloned into a p15 plasmid flanked by a pLacI constitutive promoter and the rrnB1 terminator. Ligations were made by isothermal assembly with the NEBuilder HiFi DNA assembly (NEB) using the protocol suggested by the supplier.

Libraries were transformed into E. coli DH5-alpha, grown in high volume flasks until $\mathrm{OD} \sim 1.0$ and plasmid extracted. A fraction of the grown cultures was spread on LB agar plates for colony counting and 10 colonies from each library were sequenced for the quality control. The experimental library size ranged from $2.5 \times 10^{4}$ to $8.0 \times 10^{4}$ (Additional file 1: Table S3), which is generally determined by ligation and transformation efficiency. The accuracy of the library construction, shown as percentage of perfect gene sequences, was $>60 \%$ for all the libraries (typically random indels). All the 10 sequences for each library had different nucleotides on the variant codon (Additional file 1: Table S3).

The PcaV DE libraries were transformed into electrocompetent E. coli BW25113 (K strain) cells harbouring the reporter plasmid p44pPVeGFP. The libraries 1 to 4 were combined to compose the library PcaV DE L1234 and the screening step was followed.

\section{Fluorescence activated cell sorting (FACS) counter- selection}

Cultures of E. coli BW25113 without plasmids (Negative control, C-) or transformed with plasmids containing eGFP reporter (Positive control, $\mathrm{C}+$ ); the PCA WCB system (Biosensor control); and the PcaV DE L1234 library (Tests) were grown overnight in LB media supplemented with antibiotics. Cultures were diluted (1:100) into fresh media, grown until OD 0.6 and induced with vanillin or PCA (where appropriate) for $3 \mathrm{~h}$.

Cells were centrifuged, washed with PBS and analysed using FACS (Sony SH800). Populations were sorted according to the screening method for the enrichment of mutants with altered ligand specificity. Four rounds of FACS counter-selection were made alternating negative sorting, where uninduced libraries were used to sort events on the bottom of the GFP fluorescent histogram; and positive sorting, where libraries induced with the desired effector were used to sort events on the top of the GFP fluorescent histogram.

In the 1st round, negative sorting was performed, collecting the population on the bottom $5 \%$ of the histogram. At least $2.5 \times 10^{6}$ events were passed through the flow cytometer, which correspond to $\sim 20$-fold the experimental library size $\left(1.3 \times 10^{5}\right)$, ensuring the visualization of all variants. In the 2nd round, libraries were induced with the new candidate effector vanillin at $1 \mathrm{mM}$ followed by positive sorting of the top $5 \%$ of the histogram. At least 10fold the initial library size was passed through the flow cytometer. In the 3rd round, the bottom 50\% was collected, again passing a number of events corresponding to 10 -fold the initial library size. And finally, a 4th round was performed inducing the libraries with vanillin at $1 \mathrm{mM}$ and sorting the top $5 \%$ of the histogram (Additional file 1: Figure S2). After the last round, the sorted cells were spread on LB agar plates supplemented with ampicillin $(100 \mu \mathrm{g} / \mathrm{mL})$ and chloramphenicol $(30 \mu \mathrm{g} / \mathrm{mL})$. The same procedure was performed using the parent effector PCA in parallel as a control.

A 96-well plate based screening assay was performed with 48 colonies of each sorted library testing them with the biosensor induction assay. Cultures were induced with $1.0 \mathrm{mM}$ of the corresponding inducer or water. The fluorescence was measured and the ratio induced/uninduced was used to select for responsive mutants.

\section{GC-MS analysis}

Supernatants of cultures uninduced or induced with $250 \mu \mathrm{M}, 500 \mu \mathrm{M}$ and $1 \mathrm{mM}$ of vanillin and vanillic acid were collected and frozen at $-80{ }^{\circ} \mathrm{C}$. Organic extraction was followed. $400 \mu \mathrm{L}$ of chemical standards and defrosted samples were collected and the internal standard C9 fatty acid was added to final concentration of $500 \mu \mathrm{M}$ in each one. $50 \mu \mathrm{L}$ of $5 \mathrm{M} \mathrm{HCl}$ was added and 
the tubes were vortexed for $2 \mathrm{~min}$. $400 \mu \mathrm{L}$ of methyltert-butyl ether (MTBE) was added, the tubes were vortexed again for $2 \mathrm{~min}$ and centrifuged at $11,000 \times \mathrm{g}$ for 5 min. Around $300 \mu \mathrm{L}$ of the organic layer was collected and transferred to $1.5 \mathrm{~mL}$ tubes containing small amounts of $\mathrm{MgSO}_{4}$ powder. The pellet was mixed to make sure all the water was adsorbed and centrifuged at $11,000 \times \mathrm{g}$ for $2 \mathrm{~min} .180 \mu \mathrm{L}$ of the supernatant was added to HPLC vials (Sigma) filtering it to make sure no trace of $\mathrm{MgSO}_{4}$ was present in the sample. Derivatisation was made by adding $20 \mu \mathrm{L}$ of $\mathrm{N}$,O-bis(trimethylsilyl) $+1 \%$ trimethylchlorosilane (BSTFA + 1\% TMCS) and then incubating the vials at $50{ }^{\circ} \mathrm{C}$ for $30 \mathrm{~min}$. Analysis was followed in GC-MS (7890B GC System / 5977B MSD Agilent Technologies).

\section{Sub-cloning, protein expression and purification}

Sequences encoding PcaV WT and Van2 mutant were PCR amplified from the plasmids p15pcaVWT and p15van2 (Additional file 1: Table S4) inserting a Nterminal TEV cleavage tag and cloned into a pET28a backbone in front of the His-tag using the NEBuilder HiFi DNA assembly method (NEB).

The genes were both expressed with the same conditions using E. coli BL21 (DE3) (NEB) leading to the PcaV and Van2 proteins. An overnight pre-culture of LB broth (Formedium) supplemented with Kanamycin $50 \mu \mathrm{g} / \mathrm{mL}$ was inoculated from $-80^{\circ} \mathrm{C}$ glycerol stocks. The expression cultures were prepared diluting the overnight cultures into LB media $(1: 100)$ and incubating at $37^{\circ} \mathrm{C}, 180$ RPM until OD 0.8. Induction was made with IPTG 0.5 $\mathrm{mM}$ for $20 \mathrm{~h}$ at $18^{\circ} \mathrm{C}$. Cells were harvested by centrifugation at $5000 \mathrm{RPM}, 4 \mathrm{C}$ for $1 \mathrm{~h}$.

The pellets were re-suspended with lysis buffer $(25$ $\mathrm{mM}$ Tris- $\mathrm{HCl}, 500 \mathrm{mM} \mathrm{NaCl}, 10 \mathrm{mM}$ imidazole, $\mathrm{pH}$ 7.5) supplemented with EDTA-free protease inhibitor (Roche) and $2.5 \mathrm{U} / \mathrm{mL}$ Benzonase nuclease (Sigma). Cell lysis was made using high-pressure with a cell disruptor and centrifuged at high speed $(40,000 \times \mathrm{g})$ to remove the debris. The lysate was loaded into a column of Ni-NTA Agarose beads (Qiagen) and the protein was purified using increasing concentrations of imidazole. The purified fractions were analysed on a SDS-PAGE, the cleaner fractions were mixed and dialysed.

The His-TEV tag was cleaved using TEV protease (Sigma). The reaction was made overnight at $4{ }^{\circ} \mathrm{C}$ into dialysis buffer for TEV ( $25 \mathrm{mM}$ Tris- $\mathrm{HCl}, 500 \mathrm{mM} \mathrm{NaCl}$, $10 \mathrm{mM}$ imidazole, $1 \mathrm{mM}$ DTT, $0.5 \mathrm{mM}$ EDTA, pH 8.0). The DTT and EDTA were removed immersing the cleaving reaction into dialysis buffer $(25 \mathrm{mM}$ Tris- $\mathrm{HCl}$, $500 \mathrm{mM} \mathrm{NaCl}, 10 \mathrm{mM}$ imidazole, $\mathrm{pH} 7.5$ ) for 1 hour. The TEV cleaved protein was obtained by a second $\mathrm{Ni}$ affinity purification step to remove the His-TEV peptide and the TEV protease. Further purification step was performed by size-exclusion using a Superdex 200 10/300 GL (GE Healthcare) column on an AKTA plus system. This final protein was analysed by SDS-PAGE and concentrated.

\section{Biophysical characterisation}

Freshly prepared proteins were used to analyse the biophysical properties and dimerization by size-exclusion chromatography coupled to multi-angle light scattering (SEC-MALS) analysis. The samples of PcaV and Van2 $(0.75 \mathrm{~mL}$ at $1.5 \mathrm{mg} / \mathrm{mL})$ were loaded in a size exclusion column Superdex 10/300 GL (GE Healthcare) connected to a Wyatt DAWN Heleos II EOS 18-angle laser photometer coupled to a Wyatt Optilab rEX refractive index detector. Data analysis was performed using the Astra 6 software (Wyatt technology Corp.)

Surface Plasmon Resonance (SPR) experiments were performed using the Biacore T200 system (GE Healthcare). The PcaV and Van2 proteins obtained from the SEC peak were diluted in SPR buffer $(10 \mathrm{mM}$ Hepes, $150 \mathrm{mM} \mathrm{NaCl}, \mathrm{pH}$ 7.4). The PV Bio DNA probe with a biotin tag attached to the $5^{\prime}$ end was ordered from IDT (Additional file 1: Table S1). The SPR chip GE CM5 (GE) was used with SPR buffer (10 mM Hepes, $150 \mathrm{mM}$ $\mathrm{NaCl}, \mathrm{pH}$ 7.4). In a constant flux, neutravidine was immobilised on the chip's covalent dextran matrix. Blocking of the non-bound area was made with ethanolamine present in the quenching buffer. Around $100 \mathrm{nM}$ of the 5' Bio-PV probe was used to bind the neutroavidine and cover the chip. Decreasing concentrations of PcaV or Van2 were tested to determine the affinity constant to the DNA probe. Concentrations of PcaV from $2 \mu \mathrm{g} / \mathrm{mL}$ to $240 \mu \mathrm{g} / \mathrm{mL}(0.94 \mathrm{nM}$ to $60 \mathrm{nM})$ and Van2 ranging from $20 \mu \mathrm{g} / \mathrm{mL}$ to $240 \mu \mathrm{g} / \mathrm{mL}(9.4 \mathrm{nM}$ to 600 $\mathrm{nM})$ were used.

\section{Electrophoretic mobility shift assay (EMSA)}

EMSA was performed with an infra-red based method using LI-COR EMSA Kit (LI-COR. Reactions were prepared adding stock Binding Buffer to obtain the final concentrations $(50 \mathrm{mM}$ Tris- $\mathrm{HCl}, 50 \mathrm{mM} \mathrm{KCl}, 5 \mathrm{mM}$ $\mathrm{MgCl} 2,1 \mathrm{mM}$ EDTA, 5\% (v/v) glycerol, $0.1 \mu \mathrm{g}$ poly (dI$\mathrm{dC}), 1 \mathrm{mM}$ dithiothreitol (DTT) in the reaction tube. The DNA PV probe infra-red duplex was purchased from IDT containing one palindromic sequence from the chimeric promoter $\mathrm{P}_{\mathrm{PV}}$ flanked by 6 nucleotides (32mer in total) and a $700 \mathrm{~nm}$ fluorophore tagged on the $5^{\prime}$ end of each primer (IR700-5'-TTGACTATACTC AGTGCCCTGACTATGATACT-3'). The control probe Rnd Probe IR700 duplex with the same nucleotides completely randomized was also purchased from IDT. Novex TBE - DNA retardation gels (6\%) - (ThermoFisher) were used for the electrophoresis. 
Binding assays were performed adding stocks solutions of binding buffer, nuclease-free water, DNA probe (500 $\mathrm{pM})$ and Van2 $(500 \mathrm{nM})$ or PcaV $(250 \mathrm{nM})$ proteins, according to the test order. Binding was performed by incubating the reaction tubes for $20 \mathrm{~min}$ at $30^{\circ} \mathrm{C}$. Potential ligands ( $2.5 \mathrm{mM}$ to $12.5 \mathrm{mM}$ ) were added to the binding reactions and incubated again for $30 \mathrm{~min}$ at $30^{\circ} \mathrm{C}$. The final reactions were mixed with $10 \mathrm{X}$ Orange Loading Dye (LI-COR), applied to the gel and electrophoresis was performed for $45 \mathrm{~min}$ at $100 \mathrm{~V}$. Imaging was made straight away using a $700 \mathrm{~nm}$ excitation laser in an Odyssey imager (LI-COR).

\section{Site-directed mutagenesis}

Site-directed mutagenesis was used to generate point mutation of the PcaV to Van2 aTF as well as to generate further mutants from Van2. A reverse constitutive primer (van2 PM RV - Table S1) was designed to amplify the gene downstream of the codons for amino acids 110, 113 and 114. Forward primers were designed to include one or two codon mutations keeping an overlap sequence with van2 PM RV oligo of at least 12 nucleotides (Additional file 1: Table S1). Site-directed mutagenesis PCR was made with Q5 Hot-Start High-Fidelity DNA Polymerase $(N E B)$ to amplify the p15van2 plasmid incorporating the mutations. The PCR product was ligated using the NEBuilder HiFi DNA assembly (NEB).

\section{Modelling and docking}

Homology models of Van2, and related variants, were generated with SwissModel [56] (https://swissmodel. expasy.org/) using the crystal structure of PcaV complexed with PCA (4FHT) as the template. PcaV was generated for re-docking manually deleting PCA and water molecules, except the coordinated water ( $\mathrm{HOH} 13)$, from 4FHT. All protein structures were energy minimised using either GROMOS96 force field tool inside SwissPDBViewer or Amber14SB force field inside Chimera, leading to similar outputs after docking. The effectors (PCA and vanillin) were prepared in ChemDraw and energy minimised using MM2 force field in Chem3D. Docking of PcaV (re-docking), Van2, and PcaV to Van2 mutants with PCA and vanillin was made with AutoDock Vina (version 1.1.2) [57]. The docking was performed with a flexible setting, which is based on selecting both specific amino acids in the binding-pocket and the effector to rotate during the docking. The 3 amino acid positions mutated from PcaV to Van2 (110, 113 and 114) and effectors (PCA and vanillin) were allowed to rotate. Docking results listed the 10 best conformational states in a score based on the lowest $\Delta \mathrm{G}_{\text {binding }}$ in $\mathrm{kcal} / \mathrm{mol}$.

\section{Results}

Construction and performance of the PCA biosensor

A biosensor was developed for the detection of protocatechuic acid (PCA) using the PcaV allosteric transcription factor from Streptomyces coelicolor. PcaV interacts with its DNA operator region (Oi) regulating the pcaI gene via a repression mechanism, which is abolished upon binding by hydroxyl-substituted benzoic acids, including PCA [42]. To develop the reporter component of the biosensor we employed a chimeric promoter engineering strategy [26]. Here the PcaV-Oi sequence from S. coelicolor [42] was used to design two chimeric promoter-operator sequences based on phage promoter architecture, to generate a lambda phage chimeric promoter operator $\left(\mathrm{P}_{\mathrm{LV}}\right)$, and a phage T7A1 chimeric promoter operator $\left(\mathrm{P}_{\mathrm{PV}}\right)$ [58] (Additional file 1: Figure S1A). The engineered promoters were then used to generate two biosensor designs placing each one upstream of an eGFP reporter gene. Based on this design, the reporter expression should be repressed by PcaV, and then derepressed upon PCA binding to PcaV, permitting transcription, expression of the reporter protein and generation of a fluorescent signal (Fig. 1a).

The plasmids permitting constitutive expression of PcaV (p15PcaV) and regulated expression of the eGFP reporter $\left(\mathrm{p} 44 \mathrm{P}_{\mathrm{PV}}\right.$ or $\left.\mathrm{p} 44 \mathrm{P}_{\mathrm{LV}}\right)$, which encode the biosensor system, were co-transformed into $E$. coli cells and their performance was analysed with increasing concentrations of PCA (Methods). Fitting of the dose response curves with a Hill function permitted the analysis of the biosensors performance (Additional file 1: Table S10). The $\mathrm{P}_{\mathrm{PV}}$ biosensor displayed the highest signal output, dynamic range $\left(\sim 150\right.$-fold) and greatest sensitivity $\mathrm{EC}_{50}$ of $\sim 369 \mu \mathrm{M}$, whilst the $\mathrm{P}_{\mathrm{LV}}$ biosensor showed a reduced signal, dynamic range ( $\sim 90$-fold), and sensitivity $\mathrm{EC}_{50}$ of $\sim 860 \mu \mathrm{M}$ (Fig. 1b). The best version of the PCA biosensor, the $\mathrm{P}_{\mathrm{PV}}$, was selected and used for all subsequent experiments.

Following initial performance assessment, a ligand specificity screen was conducted to identify additional effectors for the PCA biosensor. In total 29 aromatic molecules with chemical structures and functional groups, related to PCA, were selected and assessed with the PCA biosensor (Additional file 1: Table S6). The initial screening was performed with fixed concentration of each candidate effector ( $1 \mathrm{mM}$ ) (Additional file 1: Figure $\mathrm{S} 1 \mathrm{~B})$, the identified active effectors were then assessed in a full titration screen (Fig. 1c). Three active effectors were able to generate an increased output signal: protocatechuic acid or 3,4-dihydroxybenzoic acid (PCA), 3hydroxybenzoic acid (3HB) and 4-hydroxybenzoic acid (4HB), as observed by fitting the dose response curves (Additional file 1: Table S10). These three effectors contain a very similar chemical structure, with the presence 


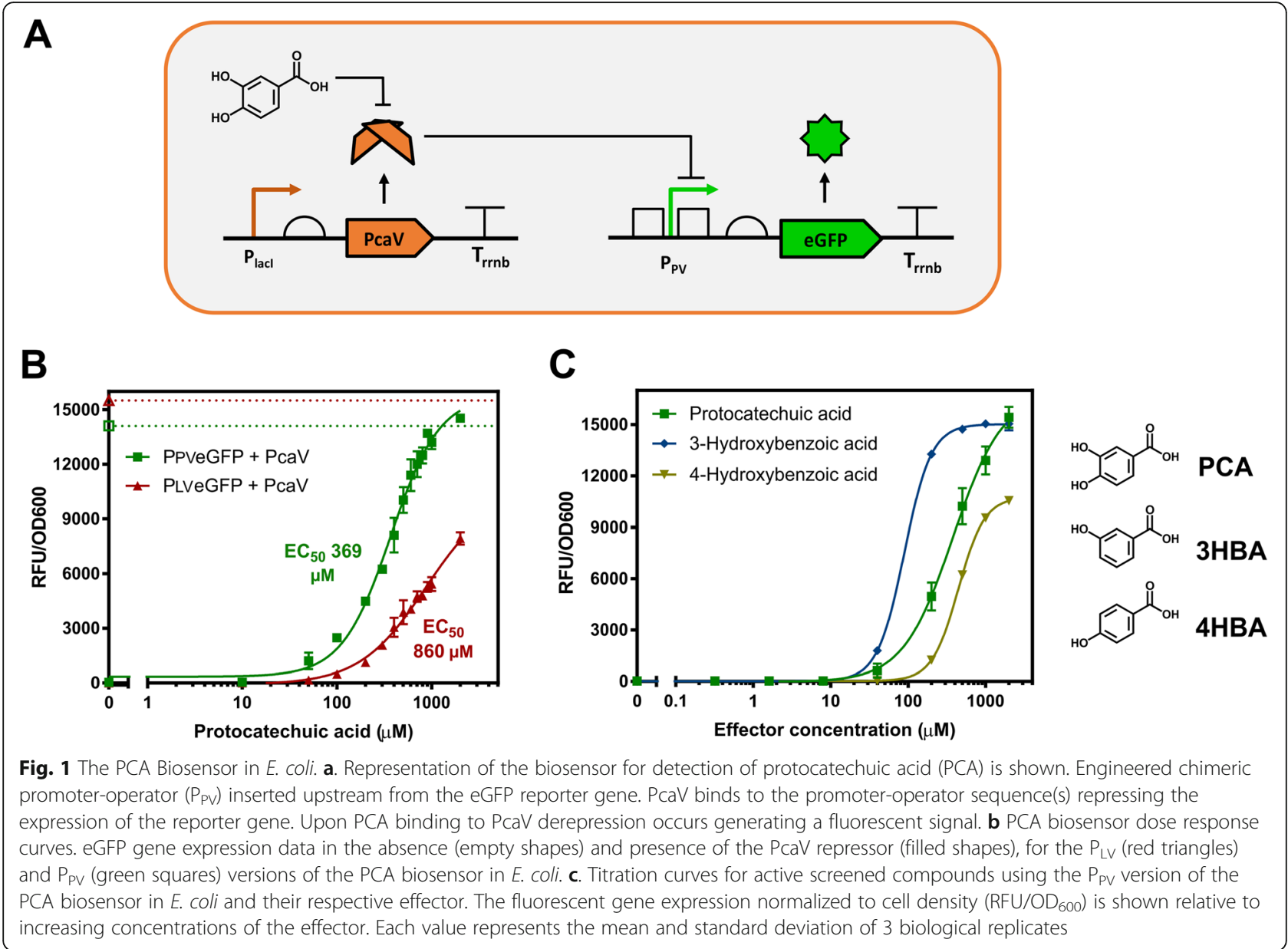

of a hydroxyl group in either the 3 or 4 position on the aromatic ring for $3 \mathrm{HB}$ and $4 \mathrm{HB}$ respectively, or both 3 and 4 for PCA. Interestingly, similar aromatic molecules did not display activity. For example, the presence of an additional hydroxyl group on the position 2 (gallic acid), presence of a methoxy group on position 3 (vanillic acid) and absence of substitutions (benzoic acid) resulted in no activity demonstrating a narrow ligand specificity pattern of the PCA biosensor (Additional file 1: Figure S1B).

The large dynamic range and narrow ligand specificity of the PCA biosensor creates sensing capabilities for the detection of hydroxyl substituted benzoic molecules, which are commonly present as intermediates in the microbial catabolic degradation pathways of lignin [59, 60]. Moreover, these performance characteristics make the PCA biosensor an ideal system to explore the potential to alter its ligand specificity using directed evolution strategies [31, 61].

\section{PcaV directed evolution}

A directed evolution strategy was employed in order to assess the plasticity of the PcaV aTF and to explore whether biosensors with altered ligand specificity could be developed from the PCA biosensor.

The structural information regarding the PcaV-PCA interaction has been previously described (PDB 4G9Y, 4FHT) [42]. Residues from both dimers interact with PCA in each binding-pocket: H21(A), W25(A), S35(A), Y38(A), A39(A), I110(A), M113(A), N114(A) from monomer $A$ and L6(B), H9(B), G11(B), H12(B), R15(B) from monomer $B$ (Fig. 2a). The amino acids on the lower part of the binding-pocket are associated with the repression mechanism, especially R15 [42]. Based on this information, a structure-based directed evolution strategy was proposed. The amino acids known to interact with the 3-hydroxy and 4-hydroxy groups, on the upper side of the effector binding-pocket, were selected for mutagenesis, whereas the amino acids on the lower side were maintained (Fig. 2a). DNA libraries encoding PcaV were designed and assembled (PcaV DE L1; L2; L3 and L4), using the NNK codon set, at positions corresponding to the 7 amino acid residues H9, H21, W25, Y38, I110, M113, N114 (Additional file 1: Table S1-2).

The PcaV DE libraries were assembled (p15 plasmid), and transformed into electrocompetent $E$. coli cells 

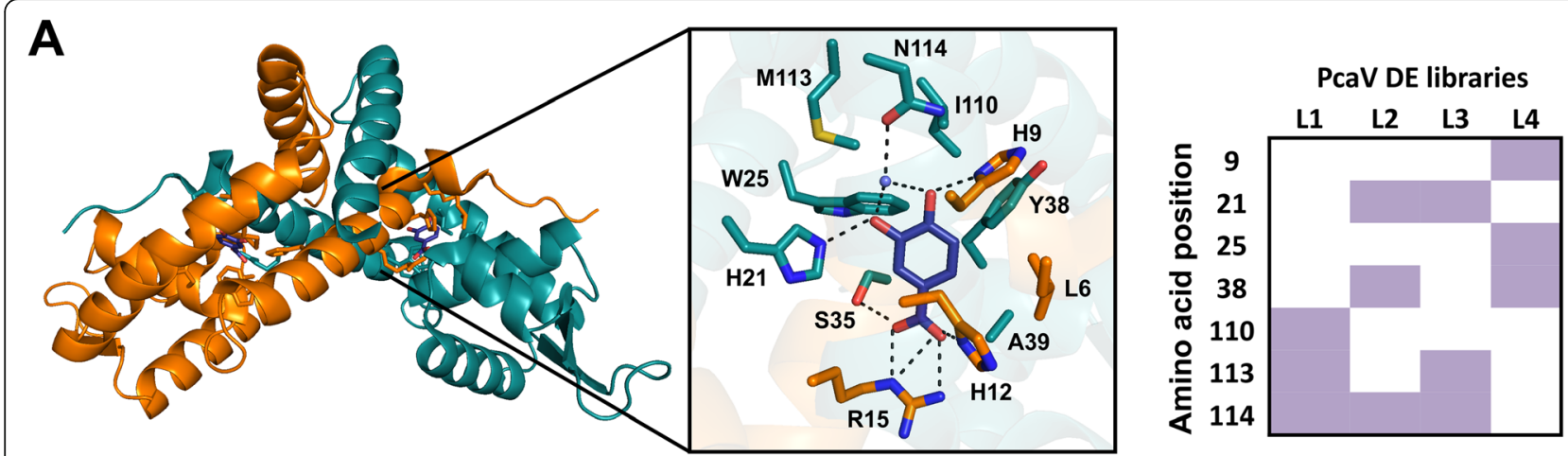

B
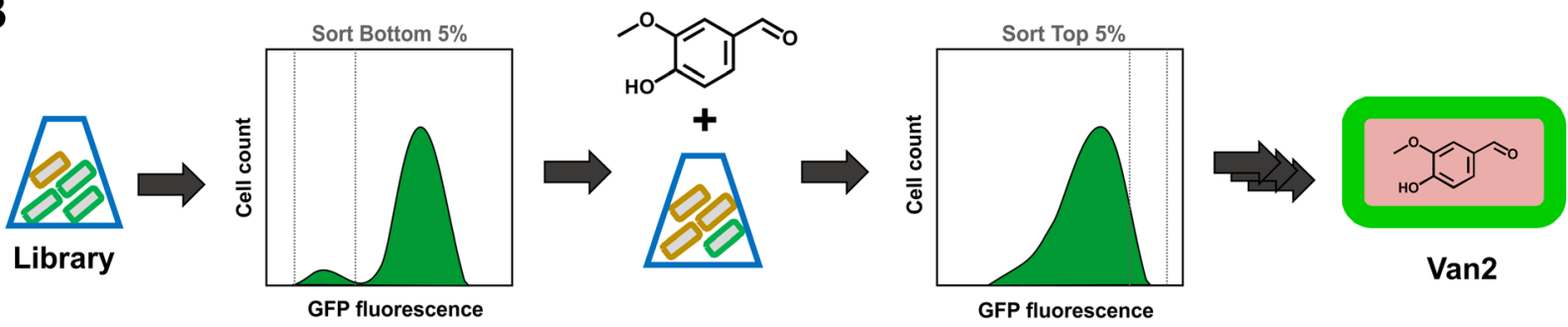

Fig. 2 Directed Evolution of PcaV. a. The amino acids H9, H21, W25, Y38, I110, M113, and N114 in the PcaV effector binding site were selected for generation of completely randomized libraries, based on the PcaV-PCA structure (PDB: 4G9Y, 4FHT) [42]. b. Cultures of E. coli with the PcaV DE Libraries were used in a Fluorescence Activated Cell Sorting (FACS) counter-selection method for the enrichment of mutants with altered ligand specificity. Rounds of FACS were made alternating between negative selection of uninduced libraries with sorting of events on the bottom of the GFP fluorescent histogram and positive selection of induced libraries with sorting of events on the top of the GFP fluorescent histogram. Platebased screening assay was performed on selected colonies from each sorted library and assessed for induction with the effector. The fluorescence was measured and the ratio induced/uninduced was used to select for responsive mutants

harbouring the reporter ( $\mathrm{p} 44 \mathrm{P}_{\mathrm{PV}}$-eGFP plasmid). The strain populations containing libraries 1 to 4 were combined and screened. Screening for functional mutants was performed using fluorescence-activated cell sorting (FACS), followed by plate screening and full titration analysis (Fig. 2b). The screening was performed to search for mutants able to detect for the industrial important chemicals, including vanillin (Table S7). To explore the potential to identify mutants with similar ligand recognition but different biosensor performance PCA was also included as a control.

The majority of variants in the mutant library were non-functional (unable to bind to the DNA) leading to eGFP expression in absence of any effectors, as observed from the population distribution in the FACS histogram for the uninduced library (Additional file 1: Figure S2B). The large number of non-functional mutants observed can be related to the allosteric regulation of PcaV, where small changes could lead to profound effects upon conformation impairing the DBD interaction with operator sequence [62]. Four rounds of FACS counter-selection were performed alternating negative and positive selection. For negative selection, the library, in the absence of candidate effectors, was sorted to select for the subpopulation with lowest fluorescence. For positive selection, the library, in the presence of candidate effectors, was sorted for the sub-population with highest fluorescence (Methods). Functional mutants were selected from the directed evolution streams of PCA and vanillin induced libraries.

\section{In vivo performance of alternative PCA biosensors}

From the PCA directed evolution screening, two mutants were identified with distinct features compared to the PcaV wild type. The mutant mutPCA1 had three amino acids substitutions (I110A, M113 L, N114S) while the mutant mutPCA2 had two amino acids substitutions (W25Y, Y38W) (Additional file 1: Figure S3A). When tested with increasing concentrations of PCA the mutPCA1 system showed a higher basal expression and reduced dynamic range compared the wild type $\mathrm{PCA}$ biosensor (Additional file 1: Figure S3A). However, when assessed against other closely related candidate ligands, an indication that its ligand specificity pattern broadened towards benzoic acid and 2-hydroxybenzoic acid was observed (Additional file 1: Figure S3B). Analysing the modified amino acids of mutPCA1, the substitution of three amino acids on the upper side of the bindingpocket, including the change of the polar amide residue asparagine to the smaller polar alcohol residue serine 
(N114S), led to increased basal expression and broader specificity. In contrast the mutPCA2 biosensor system displayed a similar performance to the parental PCA biosensor (Additional file 1: Figure S3A). Analysing the modified amino acids of mutPCA2, inversion of the parental amino acids at position 25 and 38 was well tolerated leading to small increase of the basal expression and no change in the specificity.

\section{In vivo performance of the Van2 biosensor}

From the vanillin directed evolution screening, the mutant Van2 was identified to respond to vanillin, but not the parental effector PCA (Fig. 3a). Van2 was found to have three amino acids substitutions of I110V, M113S and N114A from the PcaV WT.

Investigation of vanillin detection was made by GCMS to determine if the signal was coming from vanillin itself or a metabolic conversion product by $E$. coli. The supernatant of cells exposed to vanillin revealed a substantial metabolism of vanillin to vanillyl alcohol (Additional file 1: Figure S4A). An induction test was then made with increasing concentrations of vanillyl alcohol and no fluorescent signal was observed for the latter, validating the vanillin detection by the Van2 system (Additional file 1: Figure S4B). Aldo-keto reductases and alcohol dehydrogenases have been reported to reduce aromatic aldehydes in $E$. coli $[63,64]$. Indeed an engineered $E$. coli strain lacking all the genes for these enzymes, named RARE (reduced aromatic aldehyde reduction), was previously shown to reduce the conversion of benzaldehyde to benzyl alcohol [52]. Knockout strains of the individual genes coding for aldehyde reduction enzymes $(\triangle d k g A, \Delta d k g B, \Delta y e a E, \Delta y q h D, \Delta y a h K$ and $\triangle y j g B$ ) obtained from the Keio E. coli strain collection [65] and the RARE strain [52] were transformed with the Van2 biosensor system and tested with vanillin. All strains displayed enhanced output signal in response to vanillin compare to the wild-type, however the RARE strain had the best performance and was used for further performance characterisation of the Van2 biosensor (Additional file 1: Figure S4C).

Ligand specificity screen of the Van2 biosensor in the RARE strain was then assessed against 28 aromatic molecules, including other aldehydes, PCA and vanillic acid (Additional file 1: Table S8). The Van2 biosensor detected vanillin with dynamic range of 7.7fold and other related aromatic aldehydes (3,4-dihydroxyaldehyde, 4-hydroxy-3-methylbenzaldehyde and 2-hydroxybenzaldehyde) with dynamic range higher than 6.0 -fold at $2.5 \mathrm{mM}$ (Fig. 3b).

\section{Van2 aTF biochemical and biophysical characterisation} In order to explore the altered ligand specificity observed in vivo, a series of biochemical and biophysical analyses were conducted to characterise the Van2 aTF. PcaV and Van2 proteins were expressed and purified (Methods, Additional file 1: Figure S5A). They were analysed to determine their behaviour in solution by sizeexclusion chromatography coupled to multi-angle light scattering (SEC-MALS) (Fig. 4a). Both PcaV and Van2 were observed as monodispersed (blue and red peaks respectively) with masses of $33.5 \mathrm{kDa}$ for PcaV and 34.0 $\mathrm{kDa}$ for Van2 (blue and red strong lines inside the peaks) (Fig. 4a) indicating that they both form a homodimer in the absence of the effector, which is expected for functional aTFs [42].

The affinity of PcaV and Van2 aTFs to the palindromic DNA operator region was determined by Surface Plasmon Resonance (SPR) analysis using the DNA PV biotinylated probe (Methods, Fig. 4b). Concentrations of PcaV ranging from $0.94 \mathrm{nM}$ to $60 \mathrm{nM}$ and Van2 $9.4 \mathrm{nM}$ to $600 \mathrm{nM}$ were tested to generate sensorgrams (Additional file 1: Figure S5B). The signal was plotted and a curve was traced to calculate the affinity constants $(\mathrm{Kd})$. PcaV showed experimental affinity to the probe with a $\mathrm{Kd}$ of $5.1 \mathrm{nM}$, which is very close to the previously reported affinity for the Oi palindromic region in S. coelicolor (4.6 nM) [42]. Van2 showed a Kd of $86.7 \mathrm{nM}$, lower than the observed PcaV affinity (Fig. 4b). The lower affinity observed for Van2 compared to PcaV is also reflective of the in vivo performance of the Van2 biosensor, which shows a slightly higher basal expression (Table 1).

Electrophoretic Mobility Shift Assays (EMSA) of PcaV (Fig. 4c) and Van2 (Fig. 4d and e) were next performed with the 5 '-infrared tagged DNA PV probe in order to determine the interaction of the aTFs with the DNA operator region in the absence and presence of the effectors. PcaV formed a complex with the PV probe and this complex was disrupted in the presence of $5 \mathrm{mM}$ of PCA, but not with vanillin or vanillic acid at the same concentration (Fig. 4c). Van2 formed a complex with the PV probe, but not with the Rnd probe containing the same nucleotides as PV completely randomized (Fig. 4d). EMSA titrations with increasing concentrations of effectors, from $2.5 \mathrm{mM}$ to $12.5 \mathrm{mM}$, showed Van2-PV complex disruption for vanillin, but also PCA (Fig. 4e). The EMSA results indicate that in vitro complete derepression of PcaV-PV occurs with PCA, in contrast no derepression is observed with vanillin which is consistent with the observed in vivo biosensor performance. Van2 responds to vanillin but is still responsive to PCA in contradiction with the observed in vivo biosensor performance. The Van2 effector response pattern has changed compared to the WT PcaV, where partial derepression of the Van2-PV complex occurs. This altered response pattern is reflective of the in vivo performance of the Van2 biosensor that shows a reduced 


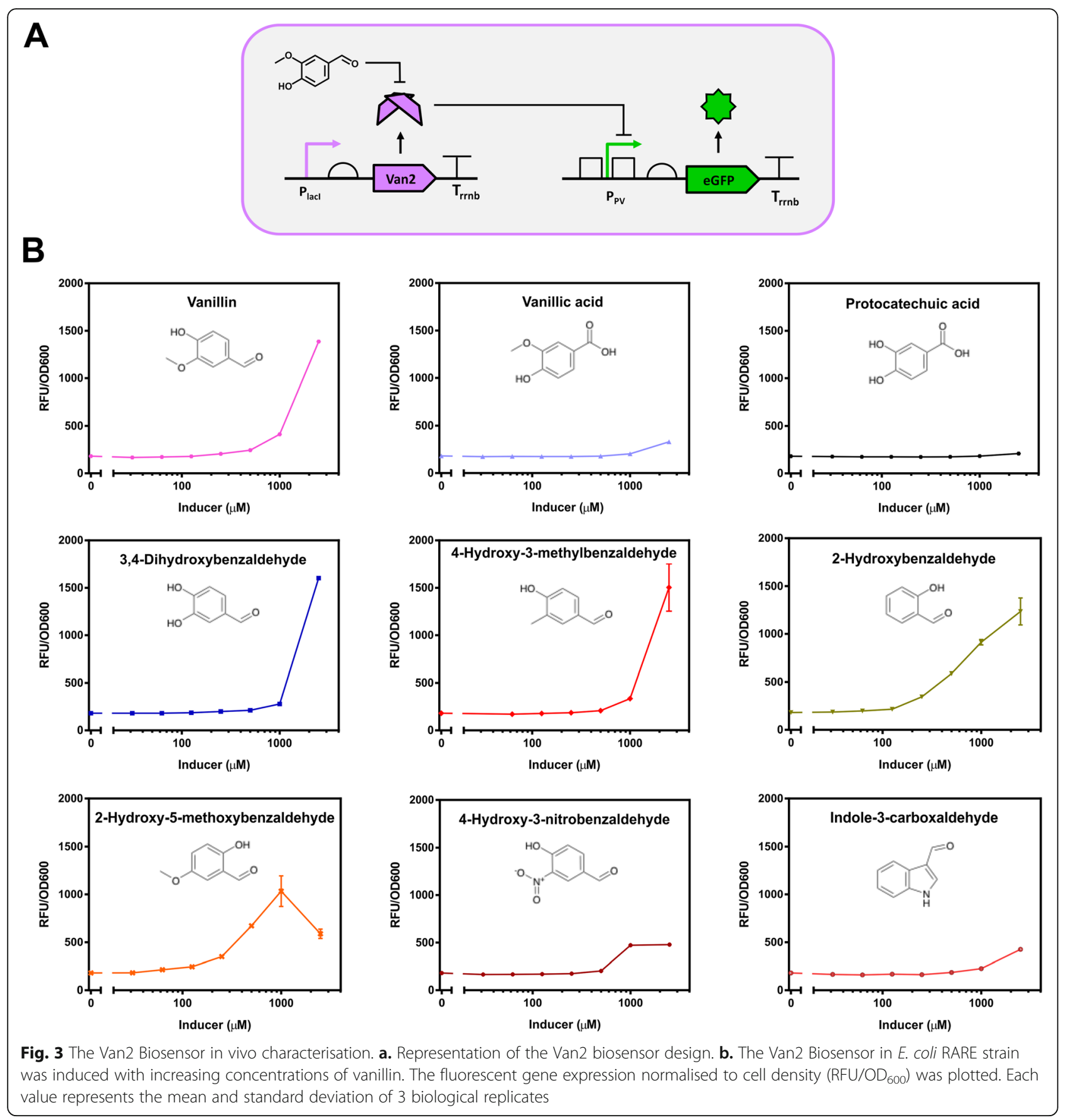

maximal expression signal for vanillin and no expression signal for PCA.

Point mutational analysis of the PcaV to Van2 mutant

In order to investigate the key amino acid substitutions responsible for the altered in vivo specificity, a strategy was employed to create and assess the point mutations of each amino acid in the mutant Van2. Intermediate mutants with one or two point mutations ranging from PcaV (I110, M113, N114) to Van2 (V110, S113, A114) were generated by site-directed mutagenesis. The mutants were tested in the biosensor system with increasing concentrations of vanillin, PCA and vanillic acid (Fig. 5a). The substitution of the amino acid I110 individually (PcaV I110V) did not affect the performance of the biosensor or the ligand specificity. The substitution of either amino acids at positions N114 or M113 individually (PcaV N114A or PcaV M113S), or of I110 in combination with N114 (PcaV I110V N114A), drastically reduced the signal output, but maintained ligand 


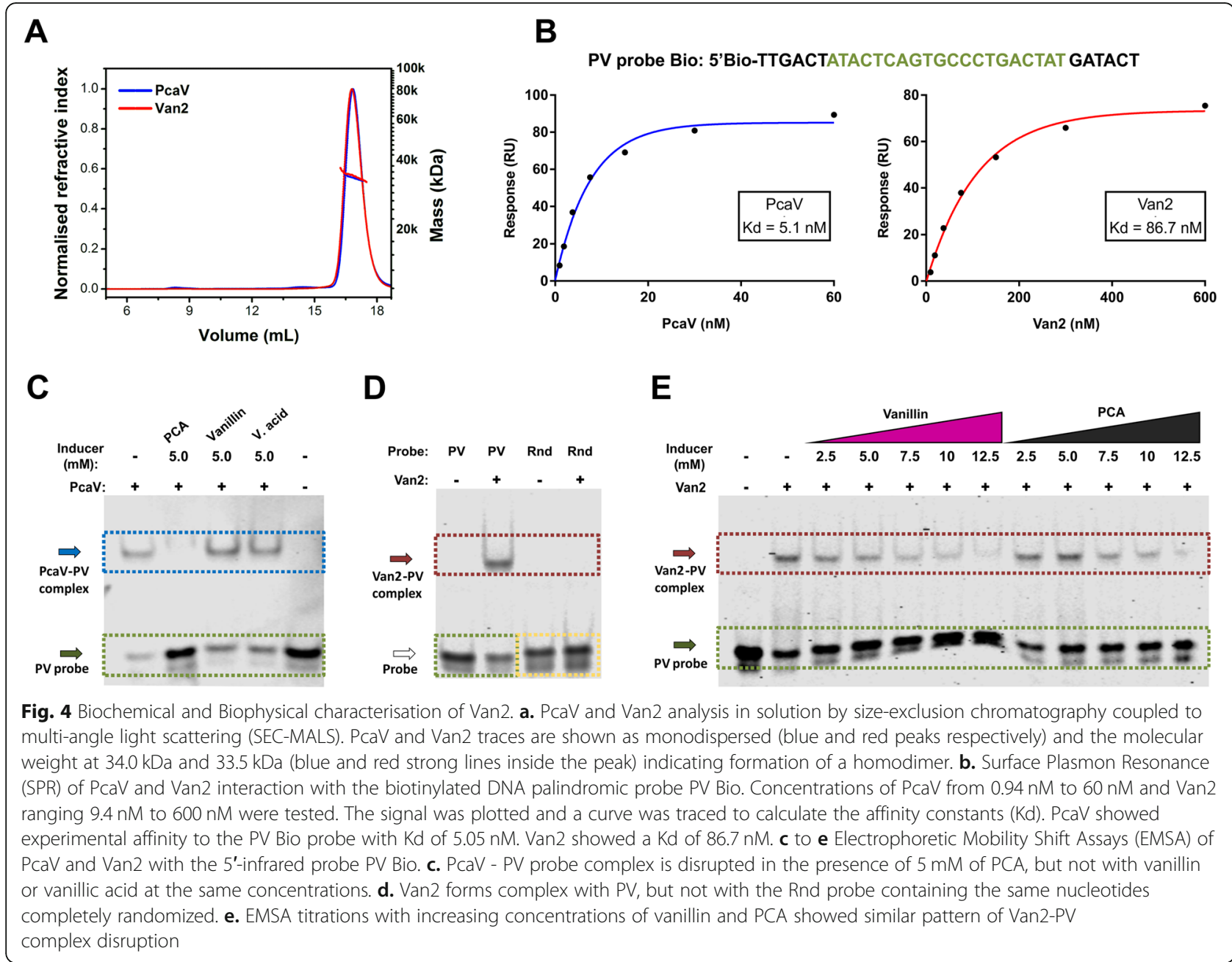

specificity toward PCA. The substitution of I110 and M113 in combination (PcaV I110V M113S) showed a small increase in basal expression and was partially responsive to all the candidate ligands assessed (vanillin, vanillic acid and PCA). The substitution of M113 and N114 in combination (PcaV M113S N114A) displayed a high basal expression indicating reduction of DNA binding affinity, but a complete change of specificity. Similar to Van2, it was responsive to vanillin, partially responsive to vanillic acid and unresponsive to PCA. The additional substitution I110V that leads to Van2 (I110V M113S N114A), conferred reduction of the basal

Table 1 Point mutational analysis of the PcaV to Van2 mutant

\begin{tabular}{|c|c|c|c|c|c|}
\hline \multirow[t]{2}{*}{ aTF } & \multirow{2}{*}{$\begin{array}{l}\text { Amino acid } \\
\text { position* }\end{array}$} & \multirow{2}{*}{$\begin{array}{l}\text { Uninduced } \\
\text { GFP/OD }\end{array}$} & \multicolumn{3}{|c|}{ Max/Min (2.5 mM induction) } \\
\hline & & & Vanillin & PCA & Vanillic acid \\
\hline PcaV & 1110; M113; N114 & $34.2 \pm 19.3$ & 1.6 & 189.9 & 2.7 \\
\hline PcaV I110V & V110; M113; N114 & $24.7 \pm 6.5$ & 1.7 & 304.4 & 8.2 \\
\hline PcaV N114A & I110; M113; A114 & $54.8 \pm 10$ & 2.5 & 18 & 2.2 \\
\hline PcaV M113S & I110; S113; N114 & $90.1 \pm 21.1$ & 3.3 & 5.1 & 4.9 \\
\hline PcaV I110V N114A & V110; M113; A114 & $74.8 \pm 7.8$ & 3.5 & 10.7 & 2.4 \\
\hline PcaV I110V M113S & V110; S113; N114 & $561.8 \pm 52.9$ & 3.3 & 2.4 & 3.9 \\
\hline PcaV M113S N114A & 1110; S113; A114 & $1830.1 \pm 60.1$ & 3.1 & 1.1 & 1.6 \\
\hline Van2 & V110; S113; A114 & $253.4 \pm 0.3$ & 5.8 & 1.2 & 2.2 \\
\hline
\end{tabular}

*Mutated amino acid positions highlighted in bold 


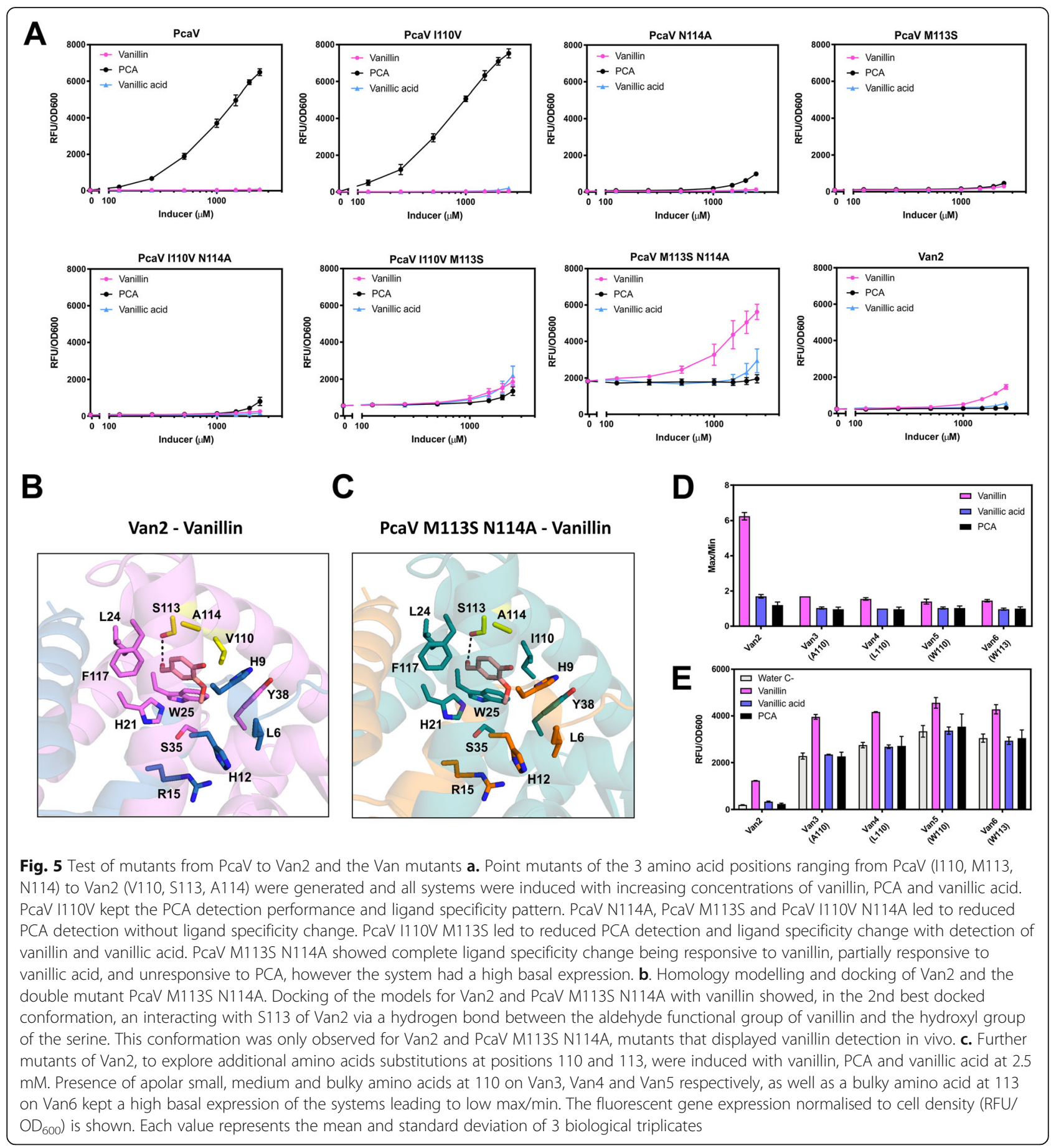

expression whilst maintaining the ligand specificity pattern. Interestingly, this same amino acid substitution in PcaV I110V had no significant effect on the performance compared to PcaV (Fig. 5a, Table 1).

\section{PcaV to Van2 modelling and docking}

Homology modelling and docking of the generated mutants were made to help understand the role of amino acid substitutions upon the altered aTF specificity.
Docking of Van2 with vanillin (Methods) led to the lowest energy conformation (Score 1: $\Delta \mathrm{G}_{\text {binding }}=-6.4 \mathrm{kcal} / \mathrm{mol}$ ) that had the same location as PCA in the PDB crystal structure (4FHT) (Additional file 1: Table S9). However interestingly, the next lowest conformation (Score 2: $\Delta \mathrm{G}_{\text {binding }}=-6.1 \mathrm{kcal} / \mathrm{mol}$ ) had vanillin in a different position interacting with S113 of Van2 via a hydrogen bond between the aldehyde functional group of vanillin and the hydroxyl group of the serine (Fig. 5b). A similar effector 
molecule orientation was also observed for the docking of PcaV double mutant M113S N114A with vanillin (Fig. 5c), consistent with the observed in vivo response (Fig. 5a). As a control re-docking of PcaV with PCA was performed, this indicated the lowest energy conformation state (Score 1: $\Delta \mathrm{G}_{\text {binding }}=-7.6 \mathrm{Kcal} / \mathrm{mol}$ ) with $\mathrm{PCA}$ positioned in the same orientation as described in the crystal structure for PcaV-PCA complex (4FHT) (Additional file 1: Figure S6). These docking conformations are consistent with the observed in vivo biosensor response. Specifically the amino acids S113 and A114 appear to play a role in the detection of vanillin by the PcaV double mutant (M113S N114A) and by Van2.

\section{Further point mutation of Van2 biosensor}

Further mutants of Van2 were designed in an attempt to more extensively explore the role and dependency of the amino acid position at 110 . The strategy was made based on the in vivo observation that S113 and A114 are essential for specificity change, but the V110 alone did not affect the aTF interaction with PCA. Three mutations were incorporated into Van2 at position 110, changing for nonpolar amino acids with increasing side chain size, generating Van3 (A110), Van4 (L110) and Van5 (W110). Additionally the Ser113 position was swapped to a nonpolar bulky residue, to generate Van6 (W113) (Table 2). The Van3-6 biosensors all displayed a high basal expression in the absence of effector, indicative of impaired DNA binding. However, this basal expression appears to be lower for the mutants with smaller amino acids (Van3 and Van4) and higher for those with bulky amino acids (Van5 and Van6) (Table 2). The overall performance for vanillin detection was lower for all the new Van aTF mutants $(<2$-fold) compared to Van2 ( $>6$-fold) (Fig. 5d, Table 2). This result indicates that V110 plays an important role in the stabilization of the Van2-DNA interaction, whilst still allowing detection of vanillin. The mechanistic basis of this interaction is not intuitive though, as the presence of other apolar amino acids such as I110 in PcaV M113S N114A, A110 in Van3 and L110 in Van4 led to high basal expression. Overall, these results indicate that the repression/derepression mechanism behind the Van2 induction by vanillin depend on the presence of the all three mutated amino acids.

\section{Discussion}

The development of new aTF-based biosensors is an important area of the synthetic biology field, as aTFs are versatile biological tools for a range of methodologies and applications [27, 28]. Developing biosensors for aromatic molecules is especially important due to their utility in industry and their availability from biomass sources [66]. Use of natural sources of sensing machinery to generate biosensors has been an approach successfully applied with a range of examples in literature $[1,18]$, however it relies on a limited set of aTFs with known inducer [6]. Therefore strategies to develop de novo aTFs for desired effectors are essential. In this sense, the directed evolution of aTFs to change the specificity patterns is an interesting strategy, regardless of the challenges associated to the allosteric nature of these proteins.

The PCA biosensor in E. coli developed here employed a heterologous aTF from Streptomyces coelicolor along with engineered chimeric promoter-operators based on phage promoter architecture. This approach yielded the highly responsive $\mathrm{P}_{\mathrm{PV}}$ version of the PCA biosensor for detection of meta, para or di-hydroxyl substituted benzoic acid. The observed large dynamic range ( $>150$-fold) is useful for selection methods based on the separation of sub-populations from genetic libraries, for example by FACS. These characteristics in conjunction with the available crystal structure of $\mathrm{PcaV}$ paved the directed evolution strategy to change the ligand specificity pattern of PcaV.

To our knowledge, this is the first work on the directed evolution of a MarR family allosteric transcription factor. In contrast to other aTF families, the MarR family has a particular structure with the DBD and EBD fused to each other [1, 45], and amino acids from different monomers are contained in the EBD [46]. A conserved scaffold is shared by this family, however the repression mechanism is variable among its members [46]. The PcaV mechanism was proposed based on the observation of apo and PcaV-PCA complex structures, where the PCA binding induces rotation of the DBD leading to

Table 2 Further point mutation of Van2 biosensor

\begin{tabular}{|c|c|c|c|c|c|c|}
\hline \multirow[t]{2}{*}{ aTF } & \multirow{2}{*}{$\begin{array}{l}\text { Amino acid } \\
\text { position* }\end{array}$} & \multirow[t]{2}{*}{ Mutated amino acid } & \multirow{2}{*}{$\begin{array}{l}\text { Uninduced } \\
\text { GFP/OD }\end{array}$} & \multicolumn{3}{|c|}{ Max/Min (2.5 mM induction) } \\
\hline & & & & Vanillin & PCA & Vanillic acid \\
\hline $\operatorname{Van} 2$ & V110; S113; A114 & $x$ & $199.4 \pm 5.9$ & 6.25 & 1.20 & 1.70 \\
\hline Van3 & A110; S113; A114 & Nonpolar small side chain & $2280.6 \pm 131.2$ & 1.70 & 0.97 & 1.03 \\
\hline Van4 & L110; S113; A114 & Nonpolar medium side chain & $2748.7 \pm 114.0$ & 1.55 & 0.97 & 1.00 \\
\hline Van5 & W110; S113; A114 & Nonpolar bulky side chain & $3339.0 \pm 249.3$ & 1.40 & 1.03 & 1.03 \\
\hline Van6 & V110; W113; A114 & Nonpolar bulky side chain & $3047.5 \pm 175.9$ & 1.45 & 1.00 & 0.97 \\
\hline
\end{tabular}

*Mutated amino acid positions highlighted in bold 
a conformation incompatible with DNA binding [42]. However, there are limited examples of MarR-DNA complex structures available [45], which is also true for PcaV [42]. Taking the allosteric nature of aTFs, this limited structural knowledge makes it even harder to predict the effect of amino acid substitutions upon the DNA binding affinity of the aTF [62]. As observed here by examining the broad fluorescence distribution of the uninduced library, it can be seen that DNA binding was affected although only amino acid positions in the EBD were targeted (Additional file 1: Figure S2B). The inherent allosteric mechanism of aTFs can hamper the search of functional mutants with the desired new property from genetic libraries $[32,62]$. High throughput methods coupled with rounds of negative (for functional DNA binding) and positive (for binding of the new effector) counter-selection have previously been employed to address these challenges by removing non-functional mutants and providing enrichment of desired mutants in the directed evolution of AraC [30, 40], PobR [33] and TrpR [34] for example. Other studies have combined high throughput screening and selective pressure methods in order to remove non-functional mutants [31, 67]. In this study, the PcaV directed evolution was performed using a FACS counter-selection method with four rounds of sorting to permit identification of the Van2 aTF.

The Van2 biosensor detected vanillin and other aromatic aldehydes in vivo without responding to the parental inducer PCA. The vanillin metabolism by the biosensor host strain was analysed by GC-MS, which showed that vanillin was partially reduced to vanillyl alcohol, but subsequent assessment of the alcohol against the biosensor indicated no detection. Furthermore, the Van2 system in the E. coli RARE strain showed the greatest signal output and dynamic range in response to vanillin, indicative of a higher availability of the effector inside the cell. In vitro a small reduction of the binding affinity of Van2 aTF to the PV DNA operator $(86.7 \mathrm{nM})$ was observed compared to PcaV $(5.05 \mathrm{nM})$ assessed by SPR, which was consistent with the higher basal expression shown in vivo for the Van2 biosensor. As observed on the EMSA assays, Van2 responds to vanillin and was still responsive to PCA in vitro. However, PcaV was not responsive to vanillin and was very responsive to PCA. The absence of an in vivo response to PCA by the Van2 biosensor could most likely be explained by physiological processes such as membrane transport selectivity affecting the intracellular availability of the effector inside the cell.

The amino acid mutations of PcaV to Van2 (I110V, M113S, N114A) led to a change in the ligand specificity. Individual mutations from the parent PcaV to Van2 indicated that the amino acids S113 and A114 have a major role on the detection of vanillin and the amino acid V110 is important to maintain repression in the absence of effector, most likely indicating that V110 is required for functional DNA binding. Interestingly, homology modelling and docking of the active in vivo mutants, Van2 and PcaV M113S N114A, with vanillin, indicated an interaction between the aldehyde functional group of vanillin and the serine at position 113. A similar observation was reported in the directed evolution of NitR to detect $\varepsilon$-caprolactam [68]. Homology modelling and docking of the NitR-L117F mutant showed a shorter distance between the effector and the mutated amino acid inside the binding-pocket compared to the WT, which suggested an association with the improved sensing [68]. Therefore the docking results for Van2 could indicate an association between the altered specificity pattern in Van2 with the availability of the position of the new effector in the binding pocket.

Other genetically encoded biosensors for aromatic aldehydes have been reported [69-74]. Among transcription factor-based biosensors, a system based on the BldR aTF of archea Sulfolobus solfataricus was developed in $E$. coli for the detection of benzaldehyde [69]. Another study used a computational based approach to reengineer the qacR aTF and generate a vanillin biosensor [70]. In other studies, biosensors based on the EmrR aTF from $E$. coli were developed allowing detection of vanillin, however these biosensors were also shown to be responsive to other aromatic acids, including vanillic and coumaric acid [71-73]. A more recent study generated a biosensor in $E$. coli based on the native YqhC aTF for the detection of aliphatic and aromatic aldehydes including glycoaldehyde and vanillin [74]. The Van2 biosensor developed here displayed high dynamic range with up to 7.7-fold signal, showed specificity for detection of vanillin and other aromatic aldehydes, and was unresponsive in vivo to the parental aromatic acid effector, PCA.

\section{Conclusion}

We report a new aTF-based biosensor to detect aromatic aldehydes, based on the reengineering of the PcaV aTF. Van2 was characterised and the role of the amino acid substitutions that led to vanillin sensing were assessed in vivo. The Van2 biosensor can detect vanillin and 3,4-dihydroxybenzaldehyde used in the flavoring industry and as chemical building blocks respectively [51, 75]. It could be applied for the improvement of biotechnological production of highvalue chemicals [52] and enable screening methodologies for discovery of enzymes able to bioprocess renewable lignin sources [71]. 
This study expands the knowledge upon the directed evolution for biosensors, specifically exploring the plasticity of a member of the MarR family of transcription factors. We demonstrate the directed evolution of the PcaV aTF to change its ligand specificity pattern and generate a biosensor for aromatic aldehydes.

\section{Supplementary information}

Supplementary information accompanies this paper at https://doi.org/10. 1186/s13036-019-0214-z.

Additional file 1: Contains supplementary Figure S1-S6 and Table S1-10.

\section{Abbreviations}

aTF: allosteric transcription factor; PCA: Protocatechuic acid; EBD: Effector binding domain; DBD: DNA binding domain; RNAP: RNA polymerase; DMSO: Dimethyl sulfoxide; RFU: Relative fluorescence unit; OE-PCR: Overlapextension polymerase chain reaction; DE: Directed evolution; FACS: Fluorescence activated cell sorting; MTBE: Methyl-tert-butyl ether; EMSA: Electrophoretic mobility shift assay; SEC-MALS: Size-exclusion chromatography coupled to multi-angle light scattering; SPR: Surface Plasmon Resonance

\section{Acknowledgments}

We would like to thank Dr. Joanne Porter from Prof. Nick Turner's group for the support with the GC-MS analysis. We would also like to thank Kate Young for the help on the Van mutants cloning steps. We would like to thank Prof. David Leys' group for the help on the protein purification and Dr. Thomas Jowitt from the Biomolecular Analysis Core Facility of the Faculty of Biology, Medicine and Health for the SEC-MALS and SPR analysis. We also would like to thank Dr. Linus Johannissen and Dr. Sam Hay for the valuable advice and discussion regarding the modelling and docking methods.

\section{Authors' contributions}

ND designed and coordinated the study. LM planned and performed the experiments. AC supported the directed evolution design. LM and ND analysed the data. LM and ND wrote the manuscript. All authors read and approved the final version of the manuscript.

\section{Funding}

ND is supported by the BBSRC David Phillips Fellowship (BB/K014773/1) and BBSRC grant (BB/P01738X/1). LM is supported via Science without Borders/ Ciências sem fronteiras scheme from the CNPq, Brazil (233608/2014-1) and the Presidential Doctoral Scheme at The University of Manchester.

\section{Availability of data and materials}

Data generated or analysed during this study are either included in this published article or are available from the corresponding author on reasonable request.

\section{Ethics approval and consent to participate} Not applicable.

\section{Consent for publication}

Not applicable.

\section{Competing interests}

The authors declare that they have no competing interests.

\section{Author details}

'Manchester Institute of Biotechnology (MIB), The University of Manchester, M1 7DN, Manchester, UK. ²Department of Chemistry, The University of Manchester, M1 7DN, Manchester, UK. ${ }^{3}$ SYNBIOCHEM, The University of Manchester, M1 7DN, Manchester, UK.
Received: 28 June 2019 Accepted: 16 October 2019

Published online: 27 November 2019

\section{References}

1. Fernandez-López R, Ruiz R, de la Cruz F, Moncalián G. Transcription factorbased biosensors enlightened by the analyte. Front Microbiol. 2015;6 JUL: 648.

2. Polizzi KM, Kontoravdi C. Genetically-encoded biosensors for monitoring cellular stress in bioprocessing. Curr Opin Biotechnol. 2015;31:50-6.

3. van Hijum SAFT, Medema MH, Kuipers OP. Mechanisms and evolution of control logic in prokaryotic transcriptional regulation. Microbiol Mol Biol Rev. 2009;73:481-509.

4. Browning DF, Busby SJW. Local and global regulation of transcription initiation in bacteria. Nat Rev Microbiol. 2016;14:638-50.

5. Mahr R, Frunzke J. Transcription factor-based biosensors in biotechnology: current state and future prospects. Appl Microbiol Biotechnol. 2016;100:79-90.

6. Libis V, Delépine B, Faulon J-L. Sensing new chemicals with bacterial transcription factors. Curr Opin Microbiol. 2016;33:105-12.

7. Eggeling L, Bott M, Marienhagen J. Novel screening methods-biosensors. Curr Opin Biotechnol. 2015;35 ii:30-ii:36

8. Chou HH, Keasling JD. Programming adaptive control to evolve increased metabolite production. Nat Commun. 2013:4:2595

9. Raman S, Rogers JK, Taylor ND, Church GM. Evolution-guided optimization of biosynthetic pathways. Proc Natl Acad Sci. 2014;111:201409523.

10. Seok JY, Yang J, Choi SJ, Lim HG, Choi UJ, Kim K-J, et al. Directed evolution of the 3-hydroxypropionic acid production pathway by engineering aldehyde dehydrogenase using a synthetic selection device. Metab Eng. 2018;47:113-20

11. Harrington LB, Jha RK, Kern TL, Schmidt EN, Canales GM, Finney KB, et al. Rapid Thermostabilization of bacillus thuringiensis Serovar Konkukian 97-27 Dehydroshikimate Dehydratase through a structure-based enzyme design and whole cell activity assay. ACS Synth Biol. 2017;6:120-9.

12. Xiong D, Lu S, Wu J, Liang C, Wang W, Wang W, et al. Improving key enzyme activity in phenylpropanoid pathway with a designed biosensor. Metab Eng. 2017;40:115-23.

13. Jha RK, Narayanan N, Pandey N, Bingen JM, Kern TL, Johnson CW, et al. Sensor-enabled alleviation of product inhibition in Chorismate pyruvateLyase. ACS Synth Biol. 2019:8:775-86.

14. Zhang F, Carothers JM, Keasling JD. Design of a dynamic sensor-regulator system for production of chemicals and fuels derived from fatty acids. Nat Biotechnol. 2012;30:354-9.

15. Liu D, Evans T, Zhang F. Applications and advances of metabolite biosensors for metabolic engineering. Metab Eng. 2015;31:35-43.

16. Xu P, Li L, Zhang F, Stephanopoulos G, Koffas M. Improving fatty acids production by engineering dynamic pathway regulation and metabolic control. Proc Natl Acad Sci. 2014;111:11299-304.

17. Xu P. Production of chemicals using dynamic control of metabolic fluxes. Curr Opin Biotechnol. 2018;53:12-9.

18. Rogers JK, Guzman CD, Taylor ND, Raman S, Anderson K, Church GM. Synthetic biosensors for precise gene control and real-time monitoring of metabolites. Nucleic Acids Res. 2015:43:7648-60.

19. Casadaban MJ. Transposition and fusion of the lac genes to selected promoters in Escherichia coli using bacteriophage lambda and mu. J Mol Biol. 1976;104:541-55.

20. Guzman LM, Belin D, Carson MJ, Beckwith J. Tight regulation, modulation, and high-level expression by vectors containing the arabinose PBAD promoter. J Bacteriol. 1995;177:4121-30.

21. Berens C, Hillen W. Gene regulation by tetracyclines. Constraints of resistance regulation in bacteria shape TetR for application in eukaryotes. Eur J Biochem. 2003;270:3109-21.

22. Dietrich JA, Shis DL, Alikhani A, Keasling JD. Transcription factor-based screens and synthetic selections for microbial small-molecule biosynthesis. ACS Synth Biol. 2013;2:47-58

23. Kim SK, Kim SH, Subhadra B, Woo S-G, Rha E, Kim S-W, et al. A genetically encoded biosensor for monitoring isoprene production in engineered Escherichia coli. ACS Synth Biol. 2018;7:2379-90.

24. Uchiyama T, Miyazaki K. Product-induced gene expression, a productresponsive reporter assay used to screen metagenomic libraries for enzymeencoding genes. Appl Environ Microbiol. 2010;76:7029-35. 
25. De Las HA, De Lorenzo V. In situ detection of aromatic compounds with biosensor Pseudomonas putida cells preserved and delivered to soil in water-soluble gelatin capsules. Anal Bioanal Chem. 2011;400:1093-104.

26. Machado LFM, Dixon N. Development and substrate specificity screening of an in vivo biosensor for the detection of biomass derived aromatic chemical building blocks. Chem Commun (Camb). 2016;52:11402-5.

27. De Paepe B, Peters G, Coussement P, Maertens J, De Mey M. Tailor-made transcriptional biosensors for optimizing microbial cell factories. J Ind Microbiol Biotechnol. 2017;44:623-45.

28. Koch M, Pandi A, Borkowski O, Cardoso Batista A, Faulon J-L. Custom-made transcriptional biosensors for metabolic engineering. Curr Opin Biotechnol. 2019:59:78-84.

29. Galvão TC, De Lorenzo V. Transcriptional regulators à la carte: engineering new effector specificities in bacterial regulatory proteins. Curr Opin Biotechnol. 2006;17:34-42.

30. Tang SY, Fazelinia H, Cirino PC. AraC regulatory protein mutants with altered effector specificity. J Am Chem Soc. 2008;130:5267-71.

31. Taylor ND, Garruss AS, Moretti R, Chan S, Arbing MA, Cascio D, et al. Engineering an allosteric transcription factor to respond to new ligands. Nat Methods. 2016;13:177-83.

32. Jha RK, Chakraborti S, Kern TL, Fox DT, Strauss CEM. Rosetta comparative modeling for library design: engineering alternative inducer specificity in a transcription factor. Proteins Struct Funct Bioinforma. 2015;83:1327-40.

33. Jha RK, Kern TL, Kim Y, Tesar C, Jedrzejczak R, Joachimiak A, et al. A microbial sensor for organophosphate hydrolysis exploiting an engineered specificity switch in a transcription factor. Nucleic Acids Res. 2016:44:8490-500

34. Ellefson JW, Ledbetter MP, Ellington AD. Directed evolution of a synthetic phylogeny of programmable Trp repressors. Nat Chem Biol. 2018;14:361-7.

35. Motlagh HN, Wrabl JO, Li J, Hilser VJ. The ensemble nature of allostery. Nature. 2014;508:331-9.

36. Wu J, Jiang $P$, Chen $W$, Xiong D, Huang $L$, Jia J, et al. Design and application of a lactulose biosensor. Sci Rep. 2017;7:45994.

37. Scholz O, Köstner M, Reich M, Gastiger S, Hillen W. Teaching TetR to recognize a new inducer. J Mol Biol. 2003;329:217-27.

38. Henssler E-M, Bertram R, Wisshak S, Hillen W. Tet repressor mutants with altered effector binding and allostery. FEBS J. 2005;272:4487-96.

39. Tang S-Y, Cirino PC. Design and application of a Mevalonate-responsive regulatory protein. Angew Chemie Int Ed. 2011;50:1084-6.

40. Tang S-Y, Qian S, Akinterinwa O, Frei CS, Gredell JA, Cirino PC. Screening for enhanced Triacetic acid lactone production by recombinant Escherichia coli expressing a designed Triacetic acid lactone reporter. J Am Chem Soc. 2013; 135:10099-103.

41. Davis JR, Sello JK. Regulation of genes in Streptomyces bacteria required for catabolism of lignin-derived aromatic compounds. Appl Microbiol Biotechnol. 2010;86:921-9.

42. Davis JR, Brown BL, Page R, Sello JK. Study of PcaV from Streptomyces coelicolor yields new insights into ligand-responsive MarR family transcription factors. Nucleic Acids Res. 2013;41:3888-900.

43. Wilkinson SP, Grove A. Ligand-responsive transcriptional regulation by members of the MarR family of winged helix proteins. Curr Issues Mol Biol. 2006;8:51-62.

44. Grove A. Regulation of metabolic pathways by MarR family transcription factors; 2017.

45. Perera IC, Grove A. Molecular mechanisms of ligand-mediated attenuation of DNA binding by MarR family transcriptional regulators. J Mol Cell Biol. 2010;2:243-54.

46. Deochand DK, Grove A. MarR family transcription factors: dynamic variations on a common scaffold. Crit Rev Biochem Mol Biol. 2017;52:595-613.

47. Surburg DH, Panten DJ. Individual fragrance and flavor materials. In: Common fragrance and flavor materials. Weinheim, FRG: Wiley-VCH Verlag GmbH \& Co. KGaA; 2006. p. 7-175.

48. Havkin-Frenkel D, Belanger FC. Biotechnological production of vanillin. In: Biotechnology in Flavor Production. Chichester, UK: Wiley; 2016. p. 165-192.

49. Fache M, Boutevin B, Caillol S. Vanillin production from lignin and its use as a renewable chemical. ACS Sustain Chem Eng. 2016:4:35-46.

50. Check HE. Synthetic-biology firms shift focus. Nature. 2014;505:598

51. Kunjapur AM, Prather KLJ. Microbial engineering for aldehyde synthesis. Appl Environ Microbiol. 2015;81:1892-901.

52. Kunjapur AM, Tarasova Y, Prather KL. Synthesis and accumulation of aromatic aldehydes in an engineered strain of Escherichia coli. J Am Chem Soc. 2014;136:11644-54.
53. Currin A, Swainston N, Day PJ, Kell DB. SpeedyGenes: an improved gene synthesis method for the efficient production of error-corrected, synthetic protein libraries for directed evolution. Protein Eng Des Sel. 2014;27:273-80.

54. Currin A, Swainston N, Day PJ, Kell DB. SpeedyGenes: Exploiting an Improved Gene Synthesis Method for the Efficient Production of Synthetic Protein Libraries for Directed Evolution. Humana Press, New York, NY; 2017. p. 63-78.

55. Swainston N, Currin A, Day PJ, Kell DB. GeneGenie: optimized oligomer design for directed evolution. Nucleic Acids Res. 2014;42:W395-400.

56. Waterhouse A, Bertoni M, Bienert S, Studer G, Tauriello G, Gumienny R, et al. SWISS-MODEL: homology modelling of protein structures and complexes. Nucleic Acids Res. 2018;46:W296-303.

57. Trott O, Olson AJ. AutoDock Vina: improving the speed and accuracy of docking with a new scoring function, efficient optimization, and multithreading. J Comput Chem. 2009;31:455-61.

58. Lutz R, Bujard $H$. Independent and tight regulation of transcriptional units in Escherichia coli via the LacR/O, the TetR/O and AraC/11-12 regulatory elements. Nucleic Acids Res. 1997;25:1203-10.

59. Bugg TDH, Ahmad M, Hardiman EM, Rahmanpour R. Pathways for degradation of lignin in bacteria and fungi. Nat Prod Rep. 2011;28:1883.

60. Johnson CW, Beckham GT. Aromatic catabolic pathway selection for optimal production of pyruvate and lactate from lignin. Metab Eng. 2015:28:240-7.

61. Jha RK, Kern TL, Fox DT, M. Strauss CE. Engineering an Acinetobacter regulon for biosensing and high-throughput enzyme screening in E. coli via flow cytometry. Nucleic Acids Res. 2014;42:8150-60.

62. Raman S, Taylor N, Genuth N, Fields S, Church GM. Engineering allostery. Trends Genet. 2014;30:521-8.

63. Ellis EM. Microbial aldo-keto reductases. FEMS Microbiol Lett. 2002;216:123-31.

64. Rodriguez GM, Atsumi S. Isobutyraldehyde production from Escherichia coli by removing aldehyde reductase activity. Microb Cell Factories. 2012;11:90.

65. Baba T, Ara T, Hasegawa M, Takai Y, Okumura Y, Baba M, et al. Construction of Escherichia coli K-12 in-frame, single-gene knockout mutants: the Keio collection. Mol Syst Biol. 2006;2:2006.0008.

66. Esposito D, Antonietti M. Redefining biorefinery: the search for unconventional building blocks for materials. Chem Soc Rev. 2015;44:5821-35.

67. Meyer AJ, Segall-Shapiro TH, Glassey E, Zhang J, Voigt CA. Escherichia coli "marionette" strains with 12 highly optimized small-molecule sensors. Nat Chem Biol. 2019;15:196-204.

68. Yeom S-J, Kim M, Kwon KK, Fu Y, Rha E, Park S-H, et al. A synthetic microbial biosensor for high-throughput screening of lactam biocatalysts. Nat Commun. 2018:9:5053.

69. Fiorentino G, Ronca R, Bartolucci S. A novel E. coli biosensor for detecting aromatic aldehydes based on a responsive inducible archaeal promoter fused to the green fluorescent protein. Appl Microbiol Biotechnol. 2009;82: 67-77.

70. De los Santos ELC, Meyerowitz JT, Mayo SL, Murray RM. Engineering Transcriptional Regulator Effector Specificity Using Computational Design and In Vitro Rapid Prototyping: Developing a Vanillin Sensor. ACS Synth Biol. 2016;5:287-95

71. Strachan $C R$, Singh R, Vanlnsberghe D, levdokymenko K, Budwill K, Mohn WW, et al. Metagenomic scaffolds enable combinatorial lignin transformation. Proc Natl Acad Sci U S A. 2014;111:10143-8.

72. Ho JCH, Pawar SV, Hallam SJ, Yadav VG. An improved whole-cell biosensor for the discovery of lignin-transforming enzymes in functional metagenomic screens. ACS Synth Biol. 2018;7:392-8.

73. Varman AM, Follenfant R, Liu F, Davis RW, Lin YK, Singh S. Hybrid phenolicinducible promoters towards construction of self-inducible systems for microbial lignin valorization. Biotechnol Biofuels. 2018;11:182.

74. Frazão CR, Maton V, François JM, Walther T. Development of a metabolite sensor for high-throughput detection of aldehydes in Escherichia Coli. Front Bioeng Biotechnol. 2018:6:118.

75. Gallage NJ, Moller BL. Vanillin-bioconversion and bioengineering of the most popular plant flavor and its de novo biosynthesis in the vanilla orchid. Mol Plant. 2015:8:40-57.

\section{Publisher's Note}

Springer Nature remains neutral with regard to jurisdictional claims in published maps and institutional affiliations. 